\title{
Profiles Combining Muscle Atrophy and Neutrophil-to-Lymphocyte Ratio Are Associated with Prognosis of Patients with Stage IV Gastric Cancer
}

\author{
Kota Shigeto ${ }^{1,2}$, Takumi Kawaguchi ${ }^{2, *}$ (D), Shunji Koya ${ }^{3}$, Keisuke Hirota ${ }^{3}$, \\ Toshimitsu Tanaka ${ }^{1,2}$, Sachiko Nagasu ${ }^{1}$, Masaru Fukahori ${ }^{1,2}$, Tomoyuki Ushijima ${ }^{1,2}$, \\ Hiroo Matsuse ${ }^{3,4}$, Keisuke Miwa ${ }^{1}$, Koji Nagafuji ${ }^{1,5}$ and Takuji Torimura ${ }^{2}$ \\ 1 Multidisciplinary Treatment Cancer Center, Kurume University Hospital, Kurume 830-0011, Japan; \\ kotashigeto10072@gmail.com (K.S.); tanaka_toshimitsu@med.kurume-u.ac.jp (T.T.); \\ shiraiwa_sachiko@med.kurume-u.ac.jp (S.N.); digdug0526@gmail.com (M.F.); \\ ushijima_tomoyuki@kurume-u.ac.jp (T.U.); miwakeisuke@gmail.com (K.M.); \\ knagafuji@med.kurume-u.ac.jp (K.N.) \\ 2 Division of Gastroenterology, Kurume University School of Medicine, Kurume 830-0011, Japan; \\ tori@med.kurume-u.ac.jp \\ 3 Division of Rehabilitation, Kurume University Hospital, Kurume 830-0011, Japan; \\ kouya_shunji@kurume-u.ac.jp (S.K.); hirota_keisuke@kurume-u.ac.jp (K.H.); \\ matsuse_hiroh@kurume-u.ac.jp (H.M.) \\ 4 Department of Orthopedics, School of Medicine, Kurume University, Kurume 830-0011, Japan \\ 5 Division of Hematology and Oncology, Department of Medicine, Kurume University School of Medicine, \\ Kurume 830-0011, Japan \\ * Correspondence: takumi@med.kurume-u.ac.jp; Tel.: +81-942-31-7627
}

Received: 21 May 2020; Accepted: 20 June 2020; Published: 24 June 2020

check for updates

\begin{abstract}
We aimed to investigate the impact of muscle atrophy and the neutrophil-to-lymphocyte ratio (NLR), a sub-clinical biomarker of inflammation and nutrition, on the prognosis of patients with unresectable advanced gastric cancer. We retrospectively enrolled 109 patients with stage IV gastric cancer (median age 69 years; female/male 22\%/78\%; median observational period 261 days). Independent factors and profiles for overall survival (OS) were determined by Cox regression analysis and decision-tree analysis, respectively. OS was calculated using the Kaplan-Meier method. The prevalence of muscle atrophy was $82.6 \%$ and the median NLR was 3.15. In Cox regression analysis, none of factors were identified as an independent factor for survival. The decision-tree analysis revealed that the most favorable prognostic profile was non-muscle atrophy (OS rate 36.8\%). The most unfavorable prognostic profile was the combination of muscle atrophy and high NLR (OS rate 19.6\%). The OS rate was significantly lower in patients with muscle atrophy and high NLR than in patients with non-muscle atrophy (1-year survival rate $28.5 \%$ vs. $54.7 \%$; log-rank test $p=0.0014$ ). In conclusion, "muscle atrophy and high NLR" was a prognostic profile for patients with stage IV gastric cancer. Thus, the assessment of muscle mass, subclinical inflammation, and malnutrition may be important for the management of patients with stage IV gastric cancer.
\end{abstract}

Keywords: malnutrition; skeletal muscle; inflammation; stomach cancer; mortality

\section{Introduction}

Gastric cancer is a common malignancy worldwide and the third leading cause of cancer-related death [1]. Advances in diagnosis, surgical management, and chemoradiation therapy have contributed to an improvement in the prognosis of patients with early and locally advanced gastric cancer $[2,3]$. 
However, approximately $35 \%$ of patients with gastric cancer have distant metastases and have progressed to stage IV at the time of diagnosis [4]. In the REGATTA clinical trial, gastrectomy followed by chemotherapy did not demonstrate any survival benefit compared with chemotherapy alone in patients with stage IV gastric cancer [5]. Therefore, palliative chemotherapy remains the main therapeutic strategy for stage IV gastric cancer [6]. Despite recent developments in chemotherapy, the prognosis of patients with stage IV gastric cancer remains unsatisfactory [7].

Various factors are involved in the prognosis of advanced cancer. Visceral adiposity and fatty liver are risk factors for poor prognosis of patients with various cancers [8,9]. Glasgow Prognostic Score is a well-established parameter of inflammation [10]. In addition, the neutrophil-to-lymphocyte ratio (NLR) is a sub-clinical biomarker of inflammation and nutrition, in which the number of neutrophils is divided by the number of lymphocytes. NLR is not solely an index of inflammation, but is also known to reflect nutritional status, as the total lymphocyte count is decreased in cases of malnutrition [11,12]. A high NLR is associated with poor overall survival in patients with various tumors [13-15]. In addition, a higher NLR has been reported to be superior to the Glasgow Prognostic Score to predict the recurrence after curative surgery for patients with Stage II or III gastric cancer [16]. A high NLR is also associated with poor prognosis in patients with resectable gastric cancer [17]. Moreover, an increase in NLR during adjuvant chemotherapy indicates a poor prognosis in patients with stage II or III gastric cancer [18] and is associated with nutritional impairment and poor prognosis in patients with stage IV gastric cancer [19]. Thus, NLR is an important index for the management of patients with gastric cancer of any stage.

Sarcopenia is the loss of skeletal muscle mass and strength $[20,21]$ and is seen in approximately $40 \%$ of patients with various types of advanced cancers [22]. Sarcopenia is associated with a poor prognosis in patients with esophageal cancer, colon cancer, and pancreatic cancer [23-25]. In patients with gastric cancer who underwent gastrectomy, sarcopenia is associated with severe post-operative complications [26,27]. Sarcopenia is also an independent risk factor associated with decreased survival in patients who undergo radical gastrectomy for gastric cancer [28]. Recently, sarcopenia has been reported as a prognostic factor for survival in patients with locally advanced gastroesophageal adenocarcinoma [29]. In addition, muscle atrophy assessed by CT is reported to predict overall survival in patients with gastric cancer who undergo gastrectomy [30]. However, the impact of muscle atrophy remains unclear in patients with stage IV gastric cancer.

The aim of this study was to investigate the impact of NLR and muscle atrophy on the prognosis of patients with stage IV gastric cancer.

\section{Materials and Methods}

\subsection{Study Design}

The purpose of this single-center retrospective observational study was to investigate the prognostic profiles for patients with stage IV gastric cancer.

\subsection{Ethics}

The study protocol conformed to the ethical guidelines of the Declaration of Helsinki, and prior approval was obtained from the institutional review board of Kurume University, Japan. An opt-out approach was used to obtain informed consent from the patients.

\subsection{Patients}

We enrolled 109 consecutive patients with stage IV gastric cancer between April 2014 and June 2019. The patients met the following inclusion criteria: (1) were 20 years of age or older; (2) had a performance status of grade 0-2; (3) were treated with systemic chemotherapy; and (4) underwent a biochemical examination and abdominal computed tomography (CT) scans. Exclusion criteria were as follows: (1) a performance status of grade 3-4; (2) severe heart, pulmonary, renal, or brain failure; 
(3) patient suffered from inflammatory, endocrine, or gastrointestinal diseases resulting in secondary sarcopenia; and (4) had other malignancies and malabsorption.

\subsection{Diagnosis, Tumor Node Metastasis (TNM) Staging, and Treatment of Gastric Cancer}

Gastric cancer was diagnosed by a pre-chemotherapeutic biopsy. The clinical stage of gastric cancer was evaluated by TNM staging based on the Japanese gastric cancer treatment guidelines of the Japanese Gastric Cancer Association [6]. Patients were treated with chemotherapy using S-1, oxaliplatin, cisplatin, 5-fluorouracil, capecitabine, and/or trastuzumab according to the Japanese gastric cancer treatment guidelines [6].

\subsection{Prevalence of Comorbidities}

Using medical records, we examined the prevalence of diabetes mellitus, hypertension, hypercholesterolemia, and hypertriglyceridemia before chemotherapy. The diagnosis of each co-morbidity was based on the published national guidelines [31-33].

\subsection{Biochemical Examinations}

Blood was obtained after overnight fasting and biochemical tests were performed. Serum levels of aspartate aminotransferase, alanine aminotransferase, lactate dehydrogenase, alkaline phosphatase, gamma-glutamyl transpeptidase, total protein, albumin, total bilirubin, total cholesterol, triglycerides, blood urea nitrogen, creatinine, estimated glomerular filtration rate, carcinoembryonic antigen, and CA19-9 were determined. We also measured fasting blood glucose, hemoglobin A1c, prothrombin activity, red blood cell count, hemoglobin level, white blood cell count, and platelet count.

\subsection{Evaluation of NLR}

Lymphocyte and neutrophil counts were obtained from the pre-chemotherapeutic blood examination and the NLR was calculated by dividing the neutrophil count by the lymphocyte count $[11,12]$. All subjects were classified into a high or low NLR group, as defined by the median value (median NLR, 3.15).

\subsection{Evaluation of Skeletal Muscle Mass}

Skeletal muscle mass was measured using CT imaging of the third lumbar vertebra, as previously described [34,35]. The CT images used for the measurement were performed for the assessment of gastric cancer before chemotherapy. Skeletal muscle mass was evaluated by the skeletal muscle index (SMI), which was calculated by normalizing the skeletal muscle area at the third lumbar vertebra by the square of the patient's height $\left(\mathrm{m}^{2}\right)[36,37]$. Intramuscular adipose tissue content was calculated as the lumbar muscle-to-fat attenuation ratio, as previously described [38]. These analyses were performed using the diagnostic software ImageJ Version 1.50 (National Institutes of Health, Bethesda, MD, USA) [39].

In this study, sex-specific cutoff values of SMI were used to define muscle atrophy. Sakurai et al.'s previously published cutoff values were $43.2 \mathrm{~cm}^{2} / \mathrm{m}^{2}$ in males and $34.6 \mathrm{~cm}^{2} / \mathrm{m}^{2}$ in females, and these values were derived from the lowest sex-specific quartile of SMI in a Japanese gastric cancer cohort [30]. All subjects were classified into a non-muscle atrophy or muscle atrophy group according to the sex-specific cutoff values.

\subsection{Evaluation of Visceral Fat Area and Hepatic Fat Content}

Visceral fat area and hepatic fat content were assessed using CT scan images obtained before chemotherapy. Visceral fat area at the umbilical level is known to significantly correlate with total visceral abdominal fat volume [40,41]. Therefore, slices at the umbilical level were used for the evaluation of visceral fat area, as previously described [38]. Visceral adiposity was diagnosed as 
$\geq 100 \mathrm{~cm}^{2}$ of visceral fat area [42]. Hepatic fat content was calculated using the liver-to-spleen ratio: liver attenuation value/spleen attenuation value. The attenuation values were the average of the four measurements in each segment of the liver and spleen, as previously described [43]. Fatty liver was diagnosed as liver-to-spleen ratios $<0.9$ [44]. These analyses were performed using the diagnostic software ImageJ Version 1.50 (National Institutes of Health, Bethesda, MD, USA) [39].

\subsection{Follow-up after Treatment with Chemotherapy for Gastric Cancer}

After treatment with chemotherapy, patients were followed-up at regular intervals by routine physical examinations, biochemical tests, $\mathrm{CT}$, and/or magnetic resonance imaging (MRI), according to the Japanese gastric cancer treatment guidelines, until death or the study censor date (12 June 2019) [6]. The median observation period was 261 days (range: 19-1348 days). All subjects were classified into the Alive or Deceased groups.

\subsection{Safety Evaluation and Assessment of Adverse Event}

Adverse events were assessed based on the National Cancer Institute Common Terminology Criteria for Adverse Events, version 4.0 [45]. In accordance with the guidelines, the dose of chemotherapy was reduced or treatment interrupted when any adverse event of grade 3 or higher severity, or any unacceptable drug-related adverse event, occurred.

\subsection{Clinical Outcome}

The primary endpoint of this study was the overall survival (OS) of the patients.

\subsection{Statistical Analysis}

Data are expressed as the median (interquartile range (IQR)), range, or frequency. The differences in continuous and categorical variables between the Alive and Deceased groups were analyzed by Wilcoxon rank sum test and chi-square test, respectively. In addition, independent factors and profiles associated with the mortality were analyzed using a Cox regression analysis with a step-wise variable selection and a decision-tree analysis, respectively [46,47]. Overall survival analysis was performed using the Kaplan-Meier method followed by a log-rank test. The level of statistical significance was set at $p<0.05$. All statistical analyses were performed using JMP ${ }^{\circledR} 14$ software (SAS Institute Inc., Cary, NC, USA).

\section{Results}

\subsection{Patients Characteristics}

The median age of study subjects was 69 years and $78.0 \%$ of subjects were male (Table 1). All subjects had stage IV gastric cancer; $68.9 \%$ of patients were HER 2 positive, and $56.9 \%$ of patients were treated with S-1/capecitabine/5-fluorouracil. Grade 3 adverse events were seen in $74.3 \%$ of patients, and neutropenia was a major adverse event, which was seen in $35.8 \%$ of patients.

Visceral adiposity and fatty liver were seen in $20 \%$ and $2 \%$ of patients. Biochemical parameters were summarized in Table S1. The median NLR was 3.15, and the prevalence of muscle atrophy was $82.6 \%$ (Table 1 ). The median survival time for all subjects was 261 days, and the 1-year, 2 -year, and 3-year survival rates were $39.1 \%, 21.6 \%$, and $7.6 \%$, respectively. 
Table 1. Patient characteristics.

\begin{tabular}{|c|c|c|c|}
\hline Variable & Reference Value & Median (IQR) & $\begin{array}{c}\text { Range } \\
\text { (Min-Max) }\end{array}$ \\
\hline Number $(n)$ & $\mathrm{N} / \mathrm{A}$ & 109 & $\mathrm{~N} / \mathrm{A}$ \\
\hline Age (Years) & $\mathrm{N} / \mathrm{A}$ & $69(65-76)$ & $55-85$ \\
\hline Sex (Female/Male) & N/A & $22.0 \% / 78.0 \%(24 / 85)$ & N/A \\
\hline Body mass index $\left(\mathrm{kg} / \mathrm{m}^{2}\right)$ & N/A & $20.34(18.7-22.6)$ & $14.9-34.8$ \\
\hline Performance status $(0 / 1 / 2 / 3 / 4)$ & N/A & $\begin{array}{c}35.8 \% / 54.1 \% / 10.1 \% / 0 \% / 0 \% \\
(39 / 59 / 11 / 0 / 0)\end{array}$ & N/A \\
\hline Gastric cancer (Initial/Recurrence) & N/A & $80.7 \% / 19.3 \%(88 / 21)$ & N/A \\
\hline Location (Cardiac/Non cardiac) & N/A & $29.4 \% / 70.6 \%(32 / 77)$ & $\mathrm{N} / \mathrm{A}$ \\
\hline HER2 (Positive/Negative/Unknown) & $\mathrm{N} / \mathrm{A}$ & $65.1 \% / 29.4 \% / 5.5 \%(71 / 32 / 6)$ & N/A \\
\hline \multicolumn{4}{|l|}{ Treatment of gastric cancer } \\
\hline SOX/SP/FOLFOX & & $19.2 \%(21 / 109)$ & \\
\hline S-1/Cape/5-FU & $\mathrm{N} / \mathrm{A}$ & $56.9 \%(62 / 109)$ & $\mathrm{N} / \mathrm{A}$ \\
\hline SOXT/SPT/CAPOXT/XPT & & $15.6 \%(17 / 109)$ & \\
\hline Others & & $8.3 \%(9 / 109)$ & \\
\hline Grade 3 adverse event (Yes/No) & & $74.3 \% / 25.7 \%(81 / 28)$ & \\
\hline Neutropenia (Yes/No) & & $35.8 \% / 64.2 \%(39 / 70)$ & \\
\hline $\begin{array}{l}\text { Pathological diagnosis of gastric cancer } \\
\text { (tub/por/sig/muc/others) }\end{array}$ & N/A & $\begin{array}{c}47.7 \% / 34.9 \% / 12.8 \% / 1.8 \% / 2.8 \% \\
(52 / 38 / 14 / 2 / 3)\end{array}$ & N/A \\
\hline Presence of distant & N/A & & N/A \\
\hline metastasis (Yes/No) & $\mathrm{N} / \mathrm{A}$ & $97.2 \% / 2.8 \%(106 / 3)$ & $\mathrm{N} / \mathrm{A}$ \\
\hline Presence of diabetes mellitus (Yes/No) & N/A & $35.8 \% / 64.2 \%(39 / 70)$ & N/A \\
\hline Presence of hypertension (Yes/No) & $\mathrm{N} / \mathrm{A}$ & $31.2 \% / 68.8 \%(34 / 75)$ & N/A \\
\hline Presence of hypercholesterolemia (Yes/No) & $\mathrm{N} / \mathrm{A}$ & $24.5 \% / 75.5 \%(26 / 80)$ & N/A \\
\hline Presence of hypertriglyceridemia (Yes/No) & $\mathrm{N} / \mathrm{A}$ & $42.0 \% / 58.0 \%(58 / 80)$ & N/A \\
\hline NLR & $0.86-2.77$ & $3.15(2.30-5.27)$ & $0.61-22.67$ \\
\hline Presence of muscle atrophy (Yes/No) & $\mathrm{N} / \mathrm{A}$ & $82.6 \% / 17.4 \% /(90 / 19)$ & $\mathrm{N} / \mathrm{A}$ \\
\hline Intramuscular adipose tissue content & $\mathrm{N} / \mathrm{A}$ & $-0.52(-0.61--0.46)$ & $-4.61--0.013$ \\
\hline Visceral fat area $\left(\mathrm{cm}^{2}\right)$ & $\mathrm{N} / \mathrm{A}$ & $53.5(26.3-92.2)$ & $4.36-237.6$ \\
\hline Visceral adiposity (Yes/No) & $<100 \mathrm{~cm}^{2}$ & $20.0 \% / 80.0 \%(20 / 82)$ & \\
\hline Liver-to-spleen ratio & $\mathrm{N} / \mathrm{A}$ & $1.20(1.092-1.34)$ & $0.83-2.16$ \\
\hline Fatty liver (Yes/No) & 0.9 & $2 \% / 98 \%(2 / 102)$ & \\
\hline
\end{tabular}

Note: Data are expressed as median (interquartile range (IQR)), range, or frequency. Abbreviations: IQR, interquartile range; N/A, not applicable; SOX, S-1 with oxaliplatin; SP, S-1 with cisplatin; FOLFOX, 5-fluorouracil with oxaliplatin; Cape, capecitabine; 5-FU, 5-fluorouracil; SOXT, S-1 with oxaliplatin and trastuzumab; SPT, S-1 with cisplatin and trastuzumab; CAPOXT, capecitabine with oxaliplatin and trastuzumab; XPT, capecitabine with cisplatin and trastuzumab; tub, tubular adenocarcinoma; por, poorly differentiated adenocarcinoma; sig, signet-ring cell carcinoma; muc, mucinous adenocarcinoma; NLR, neutrophil-to-lymphocyte ratio.

There was no significant difference in age, sex, body mass index (BMI), or performance status between those in the Alive and Deceased groups (Table 2). The prevalence of non-cardiac gastric cancer was significantly higher in the Alive group than in the Deceased group. No significant difference was seen in the chemotherapy regimen, prevalence of HER2 positive, or pathological diagnosis of gastric cancer between the Alive and Deceased groups. There was no significant difference in the prevalence of grade 3 adverse events and neutropenia between the Alive and Deceased groups.

There was no significant difference in the prevalence of visceral adiposity between the two groups. The prevalence of fatty liver was significantly higher in the Alive group compared to the Deceased group. Patients in the Deceased group had a significantly higher NLR than those in the Alive group. There was no significant difference in the prevalence of muscle atrophy and intramuscular adipose tissue content between the Alive and Deceased groups. In the Deceased group, serum albumin levels were significantly lower and blood hemoglobin A1c levels were significantly higher than those in the Alive group (Table 2). There was no significant difference between the two groups in other biochemical parameters (Table S2). 
Table 2. Comparison of patients' characteristics between the Alive and Deceased groups.

\begin{tabular}{|c|c|c|c|c|c|}
\hline \multirow{2}{*}{ Variable } & \multicolumn{2}{|c|}{ Alive } & \multicolumn{2}{|l|}{ Deceased } & \multirow[b]{2}{*}{$p$} \\
\hline & Median (IQR) & $\begin{array}{c}\text { Range } \\
\text { (Min-Max) }\end{array}$ & Median (IQR) & $\begin{array}{c}\text { Range } \\
\text { (Min-Max) }\end{array}$ & \\
\hline Number $(\mathrm{N})$ & 28 & $\mathrm{~N} / \mathrm{A}$ & 81 & $\mathrm{~N} / \mathrm{A}$ & \\
\hline Age (Years) & $71(65-76)$ & $57-82$ & $69(65-76)$ & $55-85$ & 0.4832 \\
\hline Sex (Female/Male) & $17.9 \% / 82.1 \%(5 / 23)$ & N/A & $23.5 \% / 76.5 \%(19 / 62)$ & N/A & 0.5376 \\
\hline Body mass index $\left(\mathrm{kg} / \mathrm{m}^{2}\right)$ & $20.74(18.7-22.4)$ & $15.4-25.5$ & $20.34(18.3-22.7)$ & $14.9-34.8$ & 0.8487 \\
\hline Performance status $(0 / 1 / 2 / 3 / 4)$ & $\begin{array}{c}46.4 \% / 53.6 \% / 0 \% / 0 \% / 0 \% \\
(13 / 15 / 0 / 0 / 0)\end{array}$ & $\mathrm{N} / \mathrm{A}$ & $\begin{array}{c}32.1 \% / 54.3 \% / 13.6 \% / 0 \% / 0 \% \\
(26 / 44 / 11 / 0 / 0)\end{array}$ & $\mathrm{N} / \mathrm{A}$ & 0.0821 \\
\hline Gastric cancer (Initial/Recurrence) & $\begin{array}{c}66.7 \% / 33.3 \% \\
(18 / 9)\end{array}$ & $\mathrm{N} / \mathrm{A}$ & $\begin{array}{l}85.2 \% / 14.8 \% \\
(69 / 12)\end{array}$ & N/A & 0.0652 \\
\hline Location (Cardiac/Non cardiac) & $\begin{array}{c}14.3 \% / 85.7 \% \\
(4 / 24)\end{array}$ & $\mathrm{N} / \mathrm{A}$ & $\begin{array}{l}34.6 \% / 65.4 \% \\
\quad(28 / 53)\end{array}$ & $\mathrm{N} / \mathrm{A}$ & 0.0422 \\
\hline HER2 (Positive/Negative) & $60.7 \% / 39.3 \%(17 / 11)$ & $\mathrm{N} / \mathrm{A}$ & $72 \% / 28 \%(54 / 21)$ & N/A & 0.2708 \\
\hline Treatment of gastric cancer & & & & & \\
\hline SOX/SP/FOLFOX & $67.8 \%(19)$ & & $36.7 \%(40)$ & & \\
\hline S-1/Cape/5-FU & $3.6 \%(1)$ & N/A & $18.3 \%(20)$ & N/A & 0.0882 \\
\hline SOXT/SPT/CAPOXT/XPT & $14.3 \%(4)$ & & $11.9 \%(13)$ & & \\
\hline Others & $14.3 \%(4)$ & & $8.3 \%(8)$ & & \\
\hline Grade 3 of adverse event (Yes/No) & $78.6 \% / 21.4 \%(22 / 6)$ & & $77.8 \% / 27.2 \%(59 / 22)$ & & 0.5496 \\
\hline Neutropenia (Yes/No) & $46.4 \% / 53.6 \%(13 / 15)$ & & $32.1 \% / 67.9 \%(26 / 55)$ & & 0.1727 \\
\hline Presence of diabetes mellitus (Yes/No) & $21.4 \% / 78.6 \%(6 / 22)$ & N/A & $40.7 \% / 59.3 \%(33 / 48)$ & N/A & 0.661 \\
\hline Presence of hypertension (Yes/No) & $39.3 \% / 60.7 \%(11 / 17)$ & N/A & $28.4 / \% / 71.6 \%(23 / 58)$ & $\mathrm{N} / \mathrm{A}$ & 0.2836 \\
\hline Presence of hypercholesterolemia (Yes/No) & $14.8 \% / 85.2 \%(4 / 23)$ & N/A & $27.9 \% / 72.2 \%(22 / 57)$ & N/A & 0.1742 \\
\hline Presence of hypertriglyceridemia (Yes/No) & $0 \% / 100 \%(0 / 27)$ & N/A & $9.0 \% / 91.0 \%(7 / 71)$ & $\mathrm{N} / \mathrm{A}$ & 0.1071 \\
\hline NRL & $2.52(1.87-4.38)$ & $0.61-8.29$ & $3.17(2.47-5.79)$ & $1.01-22.67$ & 0.0371 \\
\hline Presence of muscle atrophy (Yes/No) & $75.0 \% / 25.0 \%(21 / 7)$ & & $85.2 \% / 14.8 \%(69 / 12)$ & & 0.2207 \\
\hline Intramuscular adipose tissue content & $-0.54(-0.62--0.48)$ & $-1.26--0.32$ & $-0.51(-0.59--0.46)$ & $-4.61--0.013$ & 0.2539 \\
\hline Visceral fat area $\left(\mathrm{cm}^{2}\right)$ & $59.2(43.7-101.1)$ & $9.4-172.1$ & $49.1(22.2-92.2)$ & $4.36-237.6$ & 0.1674 \\
\hline Visceral adiposity (Yes/No) & $25.9 \% / 27.1 \%(7 / 20)$ & & $17.3 \% / 82.7 \%(13 / 62)$ & & 0.3985 \\
\hline Liver-to-spleen ratio & $1.20(1.08-1.35)$ & $0.83-1.46$ & $1.12(1.09-1.32)$ & $0.95-2.16$ & 0.9686 \\
\hline Fatty liver (Yes/No) & $8.0 \% / 92 \%(2 / 23)$ & & $0.0 \% / 100 \%(0 / 76)$ & & 0.0128 \\
\hline LDH(IU/L) & $183(162-263)$ & $123-499$ & $226(173-428)$ & $106-2,323$ & 0.0458 \\
\hline Albumin (g/dL) & $3.81(3.29-4.06)$ & $2.00-4.40$ & $3.47(3.10-3.70)$ & $1.80-4.85$ & 0.0313 \\
\hline $\mathrm{HbA} 1 \mathrm{c}(\%)$ & $5.7(5.4-6.0)$ & $4.9-7.4$ & $5.9(5.6-6.4)$ & $3.9-10.9$ & 0.0405 \\
\hline
\end{tabular}

Note: Data are expressed as median (interquartile range (IQR)), range, or frequency. Abbreviations: IQR, interquartile range; SOX, S-1 with oxaliplatin; SP, S-1 with cisplatin; FOLFOX, 5-fluorouracil with oxaliplatin; Cape, capecitabine; 5-FU, 5-fluorouracil; SOXT, S-1 with oxaliplatin and trastuzumab; SPT, S-1 with cisplatin and trastuzumab; CAPOXT, capecitabine with oxaliplatin and trastuzumab; XPT, capecitabine with cisplatin and trastuzumab; tub, tubular adenocarcinoma; por, poorly differentiated adenocarcinoma; sig, signet-ring cell carcinoma; muc, mucinous adenocarcinoma; AST, aspartate aminotransferase; ALT, alanine aminotransferase; LDH, lactate dehydrogenase; ALP, alkaline phosphatase; GGT, gamma-glutamyl transpeptidase; BUN, blood urea nitrogen; eGFR, estimated glomerular filtration rate; HbA1c, hemoglobin A1c; CEA, carcinoembryonic antigen; NLR, neutrophil-to-lymphocyte ratio. 


\subsection{Cox Regression Analysis with a Stepwise Variable Selection for Mortality}

NLR and muscle atrophy were selected as variables for the Cox regression analysis by a stepwise variable selection. However, these factors were not statistically associated with mortality (Table 3). Other factors including age, sex, BMI, location of gastric cancer, location of gastric cancer, treatment regimen for gastric cancer, visceral adiposity, fatty liver, serum albumin level, and blood $\mathrm{HbA1c}$ level were not selected by a stepwise variable selection. Thus, there was no single independent factor associated with mortality in this study.

Table 3. Logistic regression analysis with a stepwise variable selection for the factors associated with mortality.

\begin{tabular}{cccc}
\hline Factors & Odds Ratio & 95\% Confidence Interval & $p$ \\
\hline Muscle atrophy (Presence) & 1.8998 & $0.6596-5.4631$ & 0.2347 \\
NLR (High) & 1.4942 & $0.6247-3.5743$ & 0.3668 \\
\hline
\end{tabular}

Abbreviation: NLR, neutrophil-to-lymphocyte ratio.

\subsection{Decision-Tree Algorithm for Mortality}

A decision-tree algorithm is a data-mining technique that reveals a series of classification rules by identifying priorities, and therefore, allow us to disclose a combination of important factors for the mortality of patients. To investigate profiles associated with mortality, a decision-tree analysis was performed. The decision-tree algorithm was created using two variables and patients were classified into three groups. Muscle atrophy was the first divergence variable, and the OS rate was $36.8 \%$ in patients in the non-muscle atrophy group (Profile 1, Figure 1), compared with an OS rate of $23.3 \%$ in patients with muscle atrophy. Among patients with muscle atrophy, NLR was the second divergence variable, and the OS rate was 19.6\% in patients with muscle atrophy and high NLR (Profile 3 in Figure 1), compared to an OS of $27.2 \%$ in patients with muscle atrophy and low NLR (Profile 2, Figure 1). Other factors including age, sex, BMI, location of gastric cancer, treatment regimen for gastric cancer, visceral adiposity, and fatty liver were not identified as a divergence variable in a decision-tree analysis.

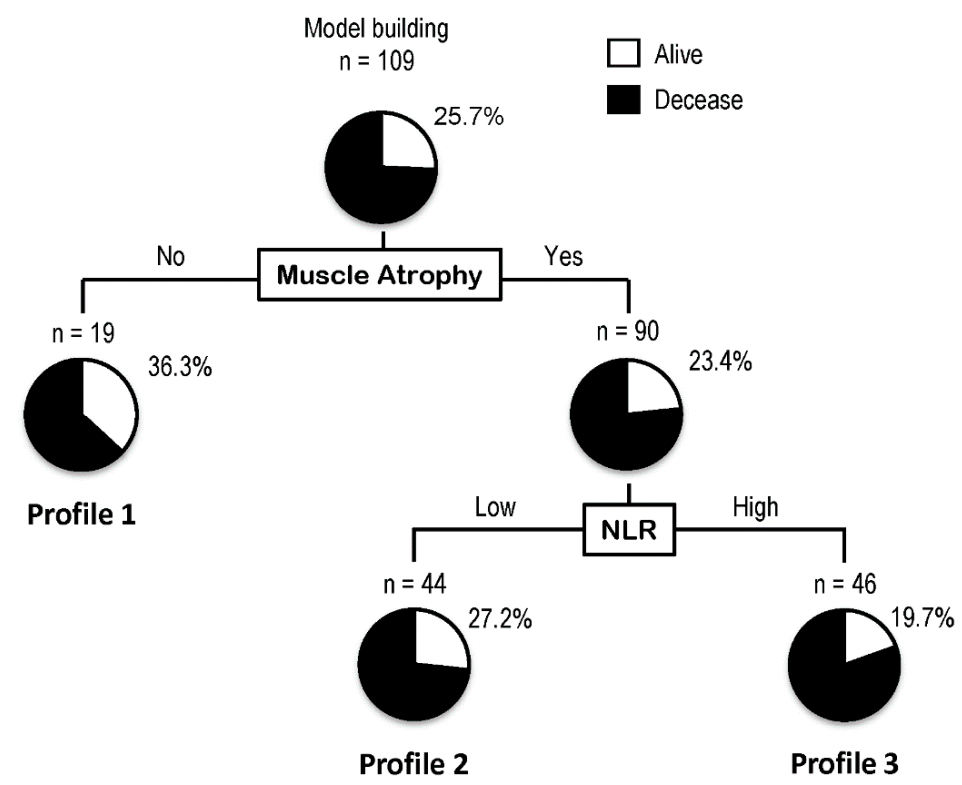

Figure 1. Profiles associated with survival in patients with stage IV gastric cancer. Decision tree algorithm for mortality. Pie graphs indicate the percentages of alive (white)/deceased (black) patients in each group. Abbreviations: NLR, neutrophil-to-lymphocyte ratio. 


\subsection{Differences in the OS among Each Profile Based on Decision-Tree Analysis for Mortality}

We further investigated the impact of profiles according to the results of decision-tree algorithm for survival. Profiles 2 and 3 were identified as independent factors for mortality in the Cox regression analysis (Profile 2, OR 2.9321, 95\%CI 1.0088-8.5220, $p=0.9481$; Profile 3, OR 6.8024, 95\%CI 1.8069-25.6093, $p=0.0046)$. Kaplan-Meier analysis was used to compare the OS rate among each profile based on the decision-tree analysis for mortality. In Profile 1, the OS rates were $54.7 \%$ and $27.3 \%$ at 1 and 2 years, respectively (Figure 2). In Profile 2, the OS rates were $43.2 \%$ and $30.5 \%$ at 1 and 2 years, respectively (Figure 2), while in Profile 3, the OS rates were $25.7 \%$ and $0.0 \%$ at 1 and 2 years, respectively (Figure 2). The OS rate of the Profile 3 was significantly lower than that of Profiles 1 and 2 (median 192 vs. 284 and 333 days, $p=0.0014$ ) (Figure 2).

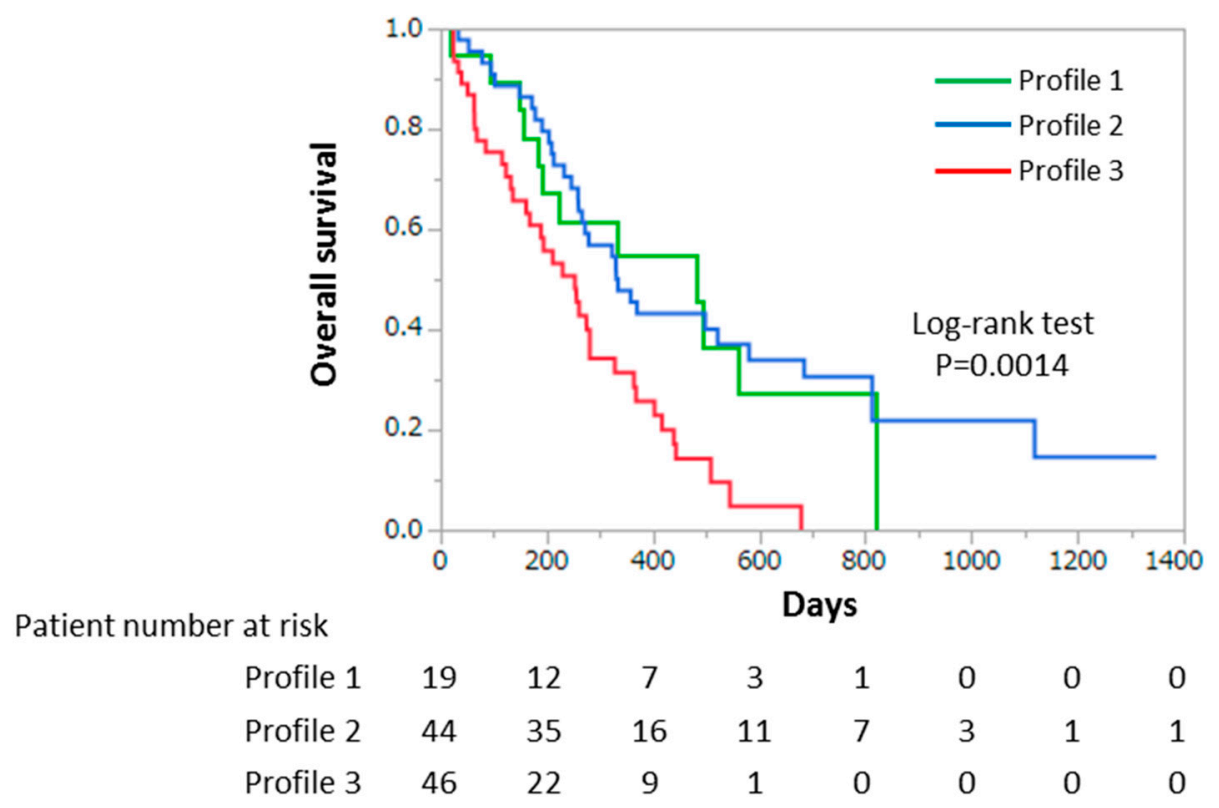

Figure 2. Overall survival time in patients with stage IV gastric cancer. Kaplan-Meier survival analysis shows the overall survival time according to Profiles 1, 2, and 3 in patients with stage IV gastric cancer. The green line represents Profile 1. The blue line represents Profile 2. The red line represents Profile 3.

\subsection{Cox Regression Analysis with a Stepwise Variable Selection for Muscle Atrophy}

We performed a Cox regression analysis and high NLR and age were identified as independent factors associated with muscle atrophy (Table 4).

Table 4. Logistic regression analysis with a stepwise variable selection for the factors associated with muscle atrophy.

\begin{tabular}{ccccc}
\hline Factors & Unit & Odds Ratio & 95\% Confidence Interval & $p$ \\
\hline NLR (High) & N/A & 1.3341 & $1.1603-1.5721$ & 0.0272 \\
Age & 1 & 1.2131 & $1.0587-1.3891$ & 0.03608 \\
\hline \multicolumn{5}{c}{ Abbreviation: NLR, neutrophil-to-lymphocyte ratio. }
\end{tabular}

\section{Discussion}

In this study, we demonstrated that "muscle atrophy combined with high NLR" was a profile associated with OS in patients with stage IV gastric cancer. The survival time was significantly shorter in patients with muscle atrophy and high NLR compared to that of patients without muscle atrophy or with muscle atrophy and low NLR. Thus, assessment for muscle mass, subclinical inflammation, and malnutrition may be important for the management of patients with advanced gastric cancer. 
In this study, the median participant age was 69 years, the median BMI was 20.3, and the median NLR was 3.15. These data are similar to those in previous studies for patients with stage IV gastric cancer $[7,48,49]$. In addition, the median survival time was 261 days in this study, comparable to the median survival time of less than 1 year that has been reported in patients with stage IV gastric cancer treated with chemotherapy $[7,49,50]$. Thus, the characteristics of patients enrolled in our study were similar to those in previous studies.

In our study, visceral adiposity and fatty liver were not identified as an independent prognostic factor. Neither NLR nor muscle atrophy were independent prognostic factors. Thus, there was no single independent variable associated with survival. However, Gonda et al. previously reported that elevated NLR is an independent prognostic factor in patients with stage IV gastric cancer [19]. Although it remains unclear why NLR was not identified as an independent prognostic factor in this study, a possible explanation is that in the previous study, $20.0 \%$ of enrolled patients showed no distant metastasis of gastric cancer [19]. However, only $2.8 \%$ of the patients enrolled in our study showed no distant metastasis. NLR has been reported to be associated with the prognosis of patients with earlier stages of gastric cancer (stage I, II, and III) rather than stage IV gastric cancer [19,51,52]. Thus, patients in our study may have a more advanced disease than those in the previous study and, therefore, various factors, rather than a single factor, are thought to be intricately associated with prognosis in this study.

No single factor was associated with mortality in this study. Therefore, a decision-tree analysis was employed to intricately assess profiles associated with survival of patients with stage IV gastric cancer. We initially identified the presence of muscle atrophy and high NLR as the primary and secondary divergence variables associated with survival, respectively. In addition, survival time was significantly shorter in patients with a profile combining muscle atrophy and high NLR than in patients with non-muscle atrophy or with muscle atrophy and low NLR. It remains unclear why muscle atrophy was identified as the most important variable for survival. It has been previously reported that muscle atrophy predicts toxicity from chemotherapy in stage IV gastric cancer patients $[53,54]$. In our study, all enrolled subjects were treated with chemotherapy. Muscle atrophy may be associated with chemotherapy-related toxicity, resulting in a poor prognosis.

In this study, a high NLR was ranked as the second prognostic variable after muscle atrophy in patients with stage IV gastric cancer. Although NLR has previously been reported as an independent prognostic factor in patients with stage IV gastric cancer [19,55,56], muscle atrophy was not evaluated in previous studies. Feliciano et al. reported that muscle atrophy combined with inflammation nearly doubled the risk of death in patients with colorectal cancer [57]. Muscle atrophy accompanied by a high NLR has also been associated with poor prognosis in patients with small cell lung cancer [58]. Thus, muscle atrophy and high NLR may synergistically worsen prognosis in patients with stage IV gastric cancer.

Any interaction between muscle atrophy and NLR remains unclear. However, high NLR, along with aging, was identified as an independent factor associated with muscle atrophy in this study. NLR is known as an indirect marker of poor nutrition status, which leads to sarcopenia [11,59]. In addition, Borges et al. recently reported that high NLR values were independently associated with sarcopenia in hospitalized patients with cancer [60]. Taken together, these data indicated a probable interaction between muscle mass and NLR.

This study has several limitations. This was a retrospective study conducted in a single center, and we enrolled patients with both initial and recurrent gastric cancer. In addition, there was heterogeneity among the enrolled subjects in the form of differing pathological diagnoses and regimens of chemotherapy. Finally, enteral nutrition was reported to improve short-term survival in patients with stage IV gastric cancer [61]; however, we did not evaluate the effects of nutritional therapy on prognosis. Thus, the prognostic importance of a profile combining muscle atrophy and high NLR should be evaluated in a multicenter prospective cohort study with a homogenous cohort of patients with stage IV gastric cancer. 


\section{Conclusions}

In conclusion, we showed that a profile combining muscle atrophy and high NLR was associated with OS in patients with stage IV gastric cancer. The prognosis was significantly worse in patients with muscle atrophy and high NLR compared to that of patients with non-muscle atrophy or with muscle atrophy and low NLR. Thus, the assessment of muscle mass, subclinical inflammation, and malnutrition may be important for the management of patients with advanced gastric cancer.

Supplementary Materials: The following are available online at http://www.mdpi.com/2072-6643/12/6/1884/s1, Table S1. Biochemical parameters or enrolled subjects; Table S2. Comparison of biochemical parameters between the Alive and Deceased groups.

Author Contributions: Conceptualization, K.S., T.K. and S.K.; Data curation, T.K., S.K., K.H., T.T. (Toshimitsu Tanaka), S.N., M.F., T.U. and K.M.; Formal analysis, T.K., S.K. and K.H.; Investigation, K.S., T.K., S.K., K.H., T.T. (Toshimitsu Tanaka), S.N., M.F., T.U. and K.M.; Methodology, T.K.; Project administration, K.S. and T.K.; Resources, T.T. (Toshimitsu Tanaka), S.N., M.F., T.U. and K.M.; Software, S.K. and K.H.; Supervision, K.N. and T.T. (Takuji Torimura); Validation, S.K., K.H., K.M., K.N. and T.T. (Takuji Torimura); Visualization, K.S., T.K. and K.H.; Writing—original draft, H.M., K.S. and T.K.; Writing—review \& editing, K.N. and T.T. (Takuji Torimura). All authors have read and agreed to the published version of the manuscript.

Funding: There was no funding for this study.

Conflicts of Interest: Takumi Kawaguchi received honoraria (lecture fees) from the Mitsubishi Tanabe Pharma Corporation, Merck Sharp and Dohme (MSD) K.K., and Otsuka Pharmaceutical Co., Ltd. The other authors declare no conflict of interest. The funders had no role in the design of the study; in the collection, analyses, or interpretation of data; in the writing of the manuscript; or in the decision to publish the results.

\section{References}

1. Ferlay, J.; Colombet, M.; Soerjomataram, I.; Mathers, C.; Parkin, D.M.; Pineros, M.; Znaor, A.; Bray, F. Estimating the global cancer incidence and mortality in 2018: GLOBOCAN sources and methods. Int. J. Cancer 2019, 144, 1941-1953. [CrossRef] [PubMed]

2. Fukunaga, S.; Nagami, Y.; Shiba, M.; Ominami, M.; Tanigawa, T.; Yamagami, H.; Tanaka, H.; Muguruma, K.; Watanabe, T.; Tominaga, K.; et al. Long-term prognosis of expanded-indication differentiated-type early gastric cancer treated with endoscopic submucosal dissection or surgery using propensity score analysis. Gastrointest Endosc. 2017, 85, 143-152. [CrossRef] [PubMed]

3. Wong, J. Effect of laparoscopic vs. open distal gastrectomy on 3-year disease free survival in patients with locally advanced gastric cancer: Commentary on the class-01 randomized clinical trial. Transl. Gastroenterol. Hepatol. 2019, 4, 78. [CrossRef] [PubMed]

4. McGhan, L.J.; Pockaj, B.A.; Gray, R.J.; Bagaria, S.P.; Wasif, N. Validation of the updated 7th edition AJCC TNM staging criteria for gastric adenocarcinoma. J. Gastrointest. Surg. 2012, 16, 53-61. [CrossRef] [PubMed]

5. Fujitani, K.; Yang, H.K.; Mizusawa, J.; Kim, Y.W.; Terashima, M.; Han, S.U.; Iwasaki, Y.; Hyung, W.J.; Takagane, A.; Park, D.J.; et al. Gastrectomy plus chemotherapy versus chemotherapy alone for advanced gastric cancer with a single non-curable factor (REGATTA): A phase 3, randomised controlled trial. Lancet Oncol. 2016, 17, 309-318. [CrossRef]

6. Japanese Gastric Cancer, A. Japanese gastric cancer treatment guidelines 2018 (5th edition). Gastric Cancer 2020. [CrossRef] [PubMed]

7. Boku, N.; Yamamoto, S.; Fukuda, H.; Shirao, K.; Doi, T.; Sawaki, A.; Koizumi, W.; Saito, H.; Yamaguchi, K.; Takiuchi, H.; et al. Fluorouracil versus combination of irinotecan plus cisplatin versus S-1 in metastatic gastric cancer: A randomised phase 3 study. Lancet Oncol. 2009, 10, 1063-1069. [CrossRef]

8. Xiao, J.; Mazurak, V.C.; Olobatuyi, T.A.; Caan, B.J.; Prado, C.M. Visceral adiposity and cancer survival: A review of imaging studies. Eur. J. Cancer Care Engl. 2018, 27, e12611. [CrossRef]

9. Hwang, Y.C.; Ahn, H.Y.; Park, S.W.; Park, C.Y. Nonalcoholic Fatty Liver Disease Associates with Increased Overall Mortality and Death from Cancer, Cardiovascular Disease, and Liver Disease in Women but Not Men. Clin. Gastroenterol. Hepatol. 2018, 16, 1131-1137.e5. [CrossRef]

10. McMillan, D.C. The systemic inflammation-based Glasgow Prognostic Score: A decade of experience in patients with cancer. Cancer Treat Rev. 2013, 39, 534-540. [CrossRef] 
11. Diaz-Martinez, J.; Campa, A.; Delgado-Enciso, I.; Hain, D.; George, F.; Huffman, F.; Baum, M. The relationship of blood neutrophil-to-lymphocyte ratio with nutrition markers and health outcomes in hemodialysis patients. Int. Urol. Nephrol. 2019, 51, 1239-1247. [CrossRef] [PubMed]

12. Forse, R.A.; Rompre, C.; Crosilla, P.; D, O.T.; Rhode, B.; Shizgal, H.M. Reliability of the total lymphocyte count as a parameter of nutrition. Can. J. Surg. 1985, 28, 216-219. [PubMed]

13. Chen, M.F.; Chen, P.T.; Kuan, F.C.; Chen, W.C. The Predictive Value of Pretreatment Neutrophil-to-Lymphocyte Ratio in Esophageal Squamous Cell Carcinoma. Ann. Surg. Oncol. 2019, 26, 190-199. [CrossRef] [PubMed]

14. Cha, Y.J.; Park, E.J.; Baik, S.H.; Lee, K.Y.; Kang, J. Clinical significance of tumor-infiltrating lymphocytes and neutrophil-to-lymphocyte ratio in patients with stage III colon cancer who underwent surgery followed by FOLFOX chemotherapy. Sci. Rep. 2019, 9, 11617. [CrossRef]

15. Aino, H.; Sumie, S.; Niizeki, T.; Kuromatsu, R.; Tajiri, N.; Nakano, M.; Satani, M.; Okamura, S.; Shimose, S.; Miyahara, K.; et al. The systemic inflammatory response as a prognostic factor for advanced hepatocellular carcinoma with extrahepatic metastasis. Mol. Clin. Oncol. 2016, 5, 83-88. [CrossRef]

16. Tanaka, H.; Tamura, T.; Toyokawa, T.; Muguruma, K.; Miki, Y.; Kubo, N.; Sakurai, K.; Hirakawa, K.; Ohira, M. Clinical Relevance of Postoperative Neutrophil-Lymphocyte Ratio (NLR) to Recurrence After Adjuvant Chemotherapy of S-1 for Gastric Cancer. Anticancer Res. 2018, 38, 3745-3751. [CrossRef]

17. Hsu, J.T.; Liao, C.K.; Le, P.H.; Chen, T.H.; Lin, C.J.; Chen, J.S.; Chiang, K.C.; Yeh, T.S. Prognostic Value of the Preoperative Neutrophil to Lymphocyte Ratio in Resectable Gastric Cancer. Medicine 2015, 94, e1589. [CrossRef]

18. Mori, M.; Shuto, K.; Kosugi, C.; Narushima, K.; Hayashi, H.; Matsubara, H.; Koda, K. An increase in the neutrophil-to-lymphocyte ratio during adjuvant chemotherapy indicates a poor prognosis in patients with stage II or III gastric cancer. BMC Cancer 2018, 18, 1261. [CrossRef]

19. Gonda, K.; Shibata, M.; Sato, Y.; Washio, M.; Takeshita, H.; Shigeta, H.; Ogura, M.; Oka, S.; Sakuramoto, S. Elevated neutrophil-to-lymphocyte ratio is associated with nutritional impairment, immune suppression, resistance to S-1 plus cisplatin, and poor prognosis in patients with stage IV gastric cancer. Mol. Clin. Oncol. 2017, 7, 1073-1078. [CrossRef]

20. Cruz-Jentoft, A.J.; Bahat, G.; Bauer, J.; Boirie, Y.; Bruyere, O.; Cederholm, T.; Cooper, C.; Landi, F.; Rolland, Y.; Sayer, A.A.; et al. Sarcopenia: Revised European consensus on definition and diagnosis. Age Ageing 2019, 48, 16-31. [CrossRef]

21. Chen, L.K.; Woo, J.; Assantachai, P.; Auyeung, T.W.; Chou, M.Y.; Iijima, K.; Jang, H.C.; Kang, L.; Kim, M.; Kim, S.; et al. Asian Working Group for Sarcopenia: 2019 Consensus Update on Sarcopenia Diagnosis and Treatment. J. Am. Med. Dir. Assoc. 2020, 21, 300-307.e2. [CrossRef] [PubMed]

22. Pamoukdjian, F.; Bouillet, T.; Levy, V.; Soussan, M.; Zelek, L.; Paillaud, E. Prevalence and predictive value of pre-therapeutic sarcopenia in cancer patients: A systematic review. Clin. Nutr. 2018, 37, 1101-1113. [CrossRef] [PubMed]

23. Onishi, S.; Tajika, M.; Tanaka, T.; Hirayama, Y.; Hara, K.; Mizuno, N.; Kuwahara, T.; Okuno, N.; Inaba, Y.; Kodaira, T.; et al. Prognostic Significance of Sarcopenia in Patients with Unresectable Advanced Esophageal Cancer. J. Clin. Med. 2019, 8, 1647. [CrossRef] [PubMed]

24. Gruber, E.S.; Jomrich, G.; Tamandl, D.; Gnant, M.; Schindl, M.; Sahora, K. Sarcopenia and sarcopenic obesity are independent adverse prognostic factors in resectable pancreatic ductal adenocarcinoma. PLoS ONE 2019, 14, e0215915. [CrossRef]

25. Choi, M.H.; Yoon, S.B.; Lee, K.; Song, M.; Lee, I.S.; Lee, M.A.; Hong, T.H.; Choi, M.G. Preoperative sarcopenia and post-operative accelerated muscle loss negatively impact survival after resection of pancreatic cancer. J. Cachexia Sarcopenia Muscle 2018, 9, 326-334. [CrossRef]

26. Zhuang, C.L.; Huang, D.D.; Pang, W.Y.; Zhou, C.J.; Wang, S.L.; Lou, N.; Ma, L.L.; Yu, Z.; Shen, X. Sarcopenia is an Independent Predictor of Severe Postoperative Complications and Long-Term Survival After Radical Gastrectomy for Gastric Cancer: Analysis from a Large-Scale Cohort. Medicine 2016, 95, e3164. [CrossRef]

27. Fukuda, Y.; Yamamoto, K.; Hirao, M.; Nishikawa, K.; Nagatsuma, Y.; Nakayama, T.; Tanikawa, S.; Maeda, S.; Uemura, M.; Miyake, M.; et al. Sarcopenia is associated with severe postoperative complications in elderly gastric cancer patients undergoing gastrectomy. Gastric Cancer 2016, 19, 986-993. [CrossRef] 
28. Zhuang, C.L.; Shen, X.; Zou, H.B.; Dong, Q.T.; Cai, H.Y.; Chen, X.L.; Yu, Z.; Wang, S.L. EWGSOP2 versus EWGSOP1 for sarcopenia to predict prognosis in patients with gastric cancer after radical gastrectomy: Analysis from a large-scale prospective study. Clin. Nutr. 2019, in press. [CrossRef]

29. Koch, C.; Reitz, C.; Schreckenbach, T.; Eichler, K.; Filmann, N.; Al-Batran, S.E.; Gotze, T.; Zeuzem, S.; Bechstein, W.O.; Kraus, T.; et al. Sarcopenia as a prognostic factor for survival in patients with locally advanced gastroesophageal adenocarcinoma. PLoS ONE 2019, 14, e0223613. [CrossRef]

30. Sakurai, K.; Kubo, N.; Tamura, T.; Toyokawa, T.; Amano, R.; Tanaka, H.; Muguruma, K.; Yashiro, M.; Maeda, K.; Hirakawa, K.; et al. Adverse Effects of Low Preoperative Skeletal Muscle Mass in Patients Undergoing Gastrectomy for Gastric Cancer. Ann. Surg. Oncol. 2017, 24, 2712-2719. [CrossRef]

31. Haneda, M.; Noda, M.; Origasa, H.; Noto, H.; Yabe, D.; Fujita, Y.; Goto, A.; Kondo, T.; Araki, E. Japanese Clinical Practice Guideline for Diabetes 2016. Diabetol. Int. 2018, 9, 1-45. [CrossRef]

32. Hoshide, S.; Kario, K.; Tomitani, N.; Kabutoya, T.; Chia, Y.C.; Park, S.; Shin, J.; Turana, Y.; Tay, J.C.; Buranakitjaroen, P.; et al. Highlights of the 2019 Japanese Society of Hypertension Guidelines and perspectives on the management of Asian hypertensive patients. J. Clin. Hypertens. Greenwich 2019, 22, 369-377. [CrossRef] [PubMed]

33. Tada, H.; Kawashiri, M.A.; Nohara, A.; Inazu, A.; Kobayashi, J.; Yasuda, K.; Mabuchi, H.; Yamagishi, M.; Hayashi, K. Lipid Management in a Japanese Community: Attainment Rate of Target Set by the Japan Atherosclerosis Society Guidelines for the Prevention of Atherosclerotic Cardiovascular Diseases 2012. J. Atheroscler. Thromb. 2017, 24, 338-345. [CrossRef] [PubMed]

34. Nagamatsu, A.; Kawaguchi, T.; Hirota, K.; Koya, S.; Tomita, M.; Hashida, R.; Kida, Y.; Narao, H.; Manako, Y.; Tanaka, D.; et al. Slow walking speed overlapped with low handgrip strength in chronic liver disease patients with hepatocellular carcinoma. Hepatol. Res. 2019, 49, 1427-1440. [CrossRef] [PubMed]

35. Hirota, K.; Kawaguchi, T.; Koya, S.; Nagamatsu, A.; Tomita, M.; Hashida, R.; Nakano, D.; Niizeki, T.; Matsuse, H.; Shiba, N.; et al. Clinical utility of the Liver Frailty Index for predicting muscle atrophy in chronic liver disease patients with hepatocellular carcinoma. Hepatol. Res. 2020, 50, 330-341. [CrossRef] [PubMed]

36. Koya, S.; Kawaguchi, T.; Hashida, R.; Goto, E.; Matsuse, H.; Saito, H.; Hirota, K.; Taira, R.; Matsushita, Y.; Imanaga, M.; et al. Effects of in-hospital exercise on liver function, physical ability, and muscle mass during treatment of hepatoma in patients with chronic liver disease. Hepatol. Res. 2017, 47, E22-E34. [CrossRef]

37. Koya, S.; Kawaguchi, T.; Hashida, R.; Hirota, K.; Bekki, M.; Goto, E.; Yamada, M.; Sugimoto, M.; Hayashi, S.; Goshima, N.; et al. Effects of in-hospital exercise on sarcopenia in hepatoma patients who underwent transcatheter arterial chemoembolization. J. Gastroenterol. Hepatol. 2019, 34, 580-588. [CrossRef]

38. Kitajima, Y.; Takahashi, H.; Akiyama, T.; Murayama, K.; Iwane, S.; Kuwashiro, T.; Tanaka, K.; Kawazoe, S.; Ono, N.; Eguchi, T.; et al. Supplementation with branched-chain amino acids ameliorates hypoalbuminemia, prevents sarcopenia, and reduces fat accumulation in the skeletal muscles of patients with liver cirrhosis. J. Gastroenterol. 2018, 53, 427-437. [CrossRef]

39. Schneider, C.A.; Rasband, W.S.; Eliceiri, K.W. NIH Image to ImageJ: 25 years of image analysis. Nat. Methods 2012, 9, 671-675. [CrossRef]

40. Tokunaga, K.; Matsuzawa, Y.; Ishikawa, K.; Tarui, S. A novel technique for the determination of body fat by computed tomography. Int. J. Obes. 1983, 7, 437-445.

41. Kvist, H.; Chowdhury, B.; Sjostrom, L.; Tylen, U.; Cederblad, A. Adipose tissue volume determination in males by computed tomography and 40 K. Int. J. Obes. 1988, 12, 249-266. [PubMed]

42. Minamino, H.; Katsushima, M.; Yoshida, T.; Hashimoto, M.; Fujita, Y.; Shirakashi, M.; Yamamoto, W.; Murakami, K.; Murata, K.; Nishitani, K.; et al. Increased circulating adiponectin is an independent disease activity marker in patients with rheumatoid arthritis: A cross-sectional study using the KURAMA database. PLoS ONE 2020, 15, e0229998. [CrossRef]

43. Endo, Y.; Ohta, M.; Tada, K.; Nakanuma, H.; Saga, K.; Masuda, T.; Hirashita, T.; Iwashita, Y.; Ozeki, Y.; Masaki, T.; et al. Improvement of non-alcoholic fatty liver disease after laparoscopic sleeve gastrectomy in Japanese obese patients. Ann. Gastroenterol. Surg. 2019, 3, 285-290. [CrossRef] [PubMed]

44. Fujii, Y.; Nanashima, A.; Hiyoshi, M.; Imamura, N.; Yano, K.; Hamada, T. Risk factors for development of nonalcoholic fatty liver disease after pancreatoduodenectomy. Ann. Gastroenterol. Surg. 2017, 1, $226-231$. [CrossRef] [PubMed] 
45. Narayan, R.; Blonquist, T.M.; Emadi, A.; Hasserjian, R.P.; Burke, M.; Lescinskas, C.; Neuberg, D.S.; Brunner, A.M.; Hobbs, G.; Hock, H.; et al. A phase 1 study of the antibody-drug conjugate brentuximab vedotin with re-induction chemotherapy in patients with CD30-expressing relapsed/refractory acute myeloid leukemia. Cancer 2020, 126, 1264-1273. [CrossRef]

46. Yamamura, S.; Kawaguchi, T.; Nakano, D.; Tomiyasu, Y.; Yoshinaga, S.; Doi, Y.; Takahashi, H.; Anzai, K.; Eguchi, Y.; Torimura, T.; et al. Profiles of advanced hepatic fibrosis evaluated by FIB-4 index and shear wave elastography in health checkup examinees. Hepatol. Res. 2020, 50, 199-213. [CrossRef] [PubMed]

47. Noda, Y.; Kawaguchi, T.; Korenaga, M.; Yoshio, S.; Komukai, S.; Nakano, M.; Niizeki, T.; Koga, H.; Kawaguchi, A.; Kanto, T.; et al. High serum interleukin-34 level is a predictor of poor prognosis in patients with non-viral hepatocellular carcinoma. Hepatol. Res. 2019, 49, 1046-1053. [CrossRef]

48. Park, J.H.; Yang, J.Y.; Park, Y.H.; Lee, W.K. The long-term prognostic difference between gastrectomy with and without preoperative chemotherapy in patients with clinical stage IV gastric cancer. Asian J. Surg. 2019, 42, 922-929. [CrossRef]

49. Ministrini, S.; Bencivenga, M.; Solaini, L.; Cipollari, C.; Sofia, S.; Marino, E.; d'Ignazio, A.; Molteni, B.; Mura, G.; Marrelli, D.; et al. Stage IV Gastric Cancer: The Surgical Perspective of the Italian Research Group on Gastric Cancer. Cancers 2020, 12, 158. [CrossRef]

50. Picado, O.; Dygert, L.; Macedo, F.I.; Franceschi, D.; Sleeman, D.; Livingstone, A.S.; Merchant, N.; Yakoub, D. The Role of Surgical Resection for Stage IV Gastric Cancer with Synchronous Hepatic Metastasis. J. Surg. Res. 2018, 232, 422-429. [CrossRef]

51. Hu, D.; Zhang, H.; Lin, X.; Chen, G.; Li, C.; Liang, B.; Chen, Y.; Cui, Z.; Peng, F.; Zheng, X.; et al. Elevated preoperative neutrophil-to-lymphocyte ratio can predict poor survival in early stage gastric cancer patients receiving radical gastrectomy: The Fujian prospective investigation of cancer (FIESTA) study. J. Cancer 2017, 8, 1214-1222. [CrossRef]

52. Shen, Q.; Liu, W.; Quan, H.; Pan, S.; Li, S.; Zhou, T.; Ouyang, Y.; Xiao, H. Prealbumin and lymphocyte-based prognostic score, a new tool for predicting long-term survival after curative resection of stage II/III gastric cancer. Br. J. Nutr. 2018, 120, 1359-1369. [CrossRef]

53. Catanese, S.; Aringhieri, G.; Cappelli, C.; Vivaldi, C.; Vitali, S.; Pecora, I.; Salani, F.; Pasquini, G.; Balducci, F.; Piercarlo, R.; et al. Baseline computed-tomography (CT)-evaluated sarcopenia predicts toxicity from first-line chemotherapy in metastatic gastric cancer (mGC) patients. Ann. Oncol. 2019, 30, iv82. [CrossRef]

54. Tan, B.H.; Brammer, K.; Randhawa, N.; Welch, N.T.; Parsons, S.L.; James, E.J.; Catton, J.A. Sarcopenia is associated with toxicity in patients undergoing neo-adjuvant chemotherapy for oesophago-gastric cancer. Eur. J. Surg. Oncol. 2015, 41, 333-338. [CrossRef] [PubMed]

55. Zhao, G.; Liu, N.; Wang, S.; Guo, J.; Song, X.; Qi, Y.; Qiu, W.; Lv, J. Prognostic significance of the neutrophil-to-lymphocyte and platelet-to-lymphocyte ratio in patients with metastatic gastric cancer. Medicine 2020, 99, e19405. [CrossRef] [PubMed]

56. Tanaka, H.; Muguruma, K.; Toyokawa, T.; Kubo, N.; Ohira, M.; Hirakawa, K. Differential impact of the neutrophil-lymphocyte ratio on the survival of patients with stage IV gastric cancer. Dig. Surg. 2014, 31, 327-333. [CrossRef]

57. Feliciano, E.M.C.; Kroenke, C.H.; Meyerhardt, J.A.; Prado, C.M.; Bradshaw, P.T.; Kwan, M.L.; Xiao, J.; Alexeeff, S.; Corley, D.; Weltzien, E.; et al. Association of Systemic Inflammation and Sarcopenia With Survival in Nonmetastatic Colorectal Cancer: Results From the C SCANS Study. JAMA Oncol. 2017, 3, e172319. [CrossRef]

58. Go, S.I.; Park, M.J.; Song, H.N.; Kang, M.H.; Park, H.J.; Jeon, K.N.; Kim, S.H.; Kim, M.J.; Kang, J.H.; Lee, G.W. Sarcopenia and inflammation are independent predictors of survival in male patients newly diagnosed with small cell lung cancer. Support. Care Cancer 2016, 24, 2075-2084. [CrossRef]

59. Sato, Y.; Gonda, K.; Harada, M.; Tanisaka, Y.; Arai, S.; Mashimo, Y.; Iwano, H.; Sato, H.; Ryozawa, S.; Takahashi, T.; et al. Increased neutrophil-to-lymphocyte ratio is a novel marker for nutrition, inflammation and chemotherapy outcome in patients with locally advanced and metastatic esophageal squamous cell carcinoma. Biomed. Rep. 2017, 7, 79-84. [CrossRef] 
60. Borges, T.C.; Gomes, T.L.; Pichard, C.; Laviano, A.; Pimentel, G.D. High neutrophil to lymphocytes ratio is associated with sarcopenia risk in hospitalized cancer patients. Clin. Nutr. 2020, in press. [CrossRef]

61. Klek, S.; Scislo, L.; Walewska, E.; Choruz, R.; Galas, A. Enriched enteral nutrition may improve short-term survival in stage IV gastric cancer patients: A randomized, controlled trial. Nutrition 2017, 36, 46-53. [CrossRef] [PubMed]

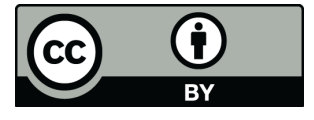

(C) 2020 by the authors. Licensee MDPI, Basel, Switzerland. This article is an open access article distributed under the terms and conditions of the Creative Commons Attribution (CC BY) license (http://creativecommons.org/licenses/by/4.0/). 